\title{
EDITORIAL AND COMMENT Reconsidering a Common Practice: In-Hospital Use of PPIs
}

\author{
Shoshana J. Herzig, MD, MPH ${ }^{1,2}$ and Robert J. Nardino, MD ${ }^{3}$ \\ 'Division of General Medicine and Primary Care, Beth Israel Deaconess Medical Center, Boston, MA, USA; ${ }^{2}$ Harvard Medical School, Boston, MA, \\ USA; ${ }^{3}$ Section of General Internal Medicine, Yale School of Medicine, New Haven, CT, USA.
}

J Gen Intern Med 31(4):361-2

DOI: $10.1007 / \mathrm{s} 11606-015-3565-2$

(c) Society of General Internal Medicine 2015

I the present issue of JGIM, Pappas et al. ${ }^{1}$ perform a simulation study with the goal of defining the net effect of proton pump inhibitor (PPI) use in hospitalized medical patients outside of the intensive care unit (ICU). Prior studies, including data from randomized, controlled trials, have demonstrated an approximately $40-60 \%$ reduction in the incidence of clinically significant gastrointestinal bleeding among hospitalized patients receiving acid-suppressive medications. ${ }^{2,3}$ However, observational studies have demonstrated associations between PPI use and pneumonia and Clostridium difficile infection in hospitalized patients. ${ }^{4,5}$ The approach by which a clinician should evaluate the balance of these benefits and risks for any given patient has remained unclear. In the present study, using existing literature-derived estimates of the associations between PPIs and upper gastrointestinal bleeding, hospital-acquired pneumonia, and Clostridium difficile infection, and the mortality associated with each condition, the authors found that PPI therapy was associated with an increase in hospital mortality in about $90 \%$ of simulated patients. This study provides novel information on the net clinical effect of PPIs in hospitalized patients outside of the ICU.

In any simulation study, the validity of the results depends largely on the validity of the estimates used to derive the simulation parameters. If these underlying estimates, which are the framework for the assumptions in the simulation, are biased in some way and fail to represent the populations from which they are drawn, then the results of the simulation will be similarly biased. Thus, a major potential limitation of this study relates to the small number of studies upon which the underlying estimates were based. Notably, the associations between PPIs and gastrointestinal bleeding and hospital-acquired pneumonia were based on only a single study each, and both were performed at the same medical center. ${ }^{2,3}$ However, tempering these limitations is the fact that even in a sensitivity analysis in which the authors assumed that the underlying probabilities of

Published online December 22, 2015 developing negative complications from PPI use and associated mortality were extremely low, and the probability of developing a gastrointestinal bleed and associated mortality was extremely high (i.e., heavily weighting the analysis towards a PPI benefit), the net effect on mortality was found to be zero, with about half of those exposed experiencing benefit, and half experiencing harm. Thus, it seems unlikely that a net benefit from PPIs would exist for the average risk patient, even if the underlying literature-derived estimates were substantially off.

Furthermore, the validity of these findings is supported by the fact that despite a significant, demonstrable benefit of acid-suppressive medications on prevention of gastrointestinal bleeding, most studies have failed to demonstrate an associated reduction in all-cause mortality, even in meta-analyses, in the setting of either prevention or treatment of gastrointestinal bleeding. ${ }^{2,6} \mathrm{Al}$ though even meta-analyses may have been underpowered for this outcome, it is notable that both reviews referenced here, and many others, have identified elevated point estimates for mortality, signaling the possibility of increased risk. These findings further support the idea that the beneficial effects of acid-suppressive medications are offset by adverse effects. Additionally, the authors in the present analysis are only able to account for adverse effects of which we are aware. The true net effect of PPIs on mortality may be shifted even further in the direction of net harm if there exist other adverse effects of PPIs, unaccounted for in the present analysis.

Although these results suggest net harm from PPIs in the majority of hospitalized patients outside the ICU, the authors appropriately note that there is a small group of patients who stand to benefit from these medications. Unfortunately, identifying patients who fall into this high-risk group is presently challenging, owing to lack of validated risk prediction models for most of the relevant clinical conditions as well as lack of widelyavailable technology capabilities for automated risk prediction embedded in electronic medical record systems. In the absence of such information and tools, the present study fills an important clinical knowledge and practice gap, suggesting that PPIs are unlikely to be of benefit for preventing gastrointestinal bleeding in the large majority of hospitalized patients, and that 
clinicians are very likely to do more harm than good by starting these agents for prophylactic purposes in the absence of other indications.

However, we would suggest that the findings related to continuation of outpatient PPIs be interpreted and acted upon with caution. The estimates used to inform the chronic use scenarios were derived from studies in the outpatient setting, and do not truly capture the inpatient risks and potential benefits of PPIs in this specific scenario. ${ }^{7,8}$ It is also often not possible for inpatient providers to discern the reasons for PPI use in the outpatient setting, and discontinuation of a PPI in a patient with a recent gastrointestinal hemorrhage could have serious consequences. Furthermore, there are other possible negative downstream effects of PPI discontinuation, including possible rebound symptoms as noted by the study authors, as well as the potential to confound the diagnosis of other chest pain syndromes arising during hospitalization (potentially leading to unnecessary diagnostic testing). That said, hospitalization may indeed provide an opportunity to discontinue these medications in a monitored setting, and is increasingly being viewed by primary care providers (PCPs) and hospitalists as an opportunity for medication reconciliation and paring down of medications lists. ${ }^{9,10}$ If this approach is taken, communication with relevant outpatient providers, including PCPs and gastroenterologists where applicable, is of paramount importance.

We applaud the authors for taking the first step in bridging the information gap that exists regarding PPI risks and benefits. For patients hospitalized in non-ICU settings who are not already taking PPIs, clinicians should carefully reconsider prescribing PPIs for ulcer prophylaxis. More data are necessary to determine if continuing outpatient PPIs in the hospital truly confers more risk than benefit, as posited by Pappas and colleagues. Until validated risk assessment tools are available to identify high risk subgroups for whom the use of PPIs would result in more benefit than harm, less is more when it comes to PPI prescribing for ulcer prophylaxis outside the ICU.

Corresponding Author: Shoshana J. Herzig, MD, MPH; 1309 Beacon Street, 2nd Floor, Brookline, MA 02446, USA (e-mail: sherzig@bidmc.harvard.edu).

\section{Compliance with Ethical Standards:}

Funding: Dr. Herzig was funded by grant number K23AG042459 from the National Institute on Aging.

Conflict of Interest: Neither of the authors have any conflicts to disclose.

\section{REFERENCES}

1. Pappas M, Jolly S, Vijan S. Defining appropriate use of proton-pump inhibitors among medical inpatients. J Gen Intern Med. doi: 10.1007/ s11606-015-3536-7.

2. Cook DJ, Reeve BK, Guyatt GH, Heyland DK, Griffith LE, Buckingham L, et al. Stress ulcer prophylaxis in critically ill patients. Resolving discordant meta-analyses. JAMA. 1996;275(4):308-314.

3. Herzig SJ, Vaughn BP, Howell MD, Ngo LH, Marcantonio ER. Acidsuppressive medication use and the risk for nosocomial gastrointestinal tract bleeding. Arch Intern Med. 2011;171(11):991-997.

4. Herzig SJ, Howell MD, Ngo LH, Marcantonio ER. Acid-suppressive medication Use and the risk for hospital-acquired pneumonia. JAMA. 2009;301(20):2120-2128.

5. Howell MD, Novack V, Grgurich $\mathbf{P}$, et al. Iatrogenic gastric acid suppression and the risk of nosocomial Clostridium difficile infection. Arch Intern Med. 2010;170(9):784-790.

6. Leontiadis GI, Sharma VK, Howden CW. Proton pump inhibitor treatment for acute peptic ulcer bleeding. Cochrane Database Syst Rev. 2006; 1:CD002094.

7. Sarkar M, Hennessy S, Yang Y-X. Proton-pump inhibitor use and the risk for community-acquired pneumonia. Ann Intern Med. 2008;149(6):391-398.

8. Gulmez SE, Holm A, Frederiksen H, Jensen TG, Pedersen C, Hallas J. Use of proton pump inhibitors and the risk of community-acquired pneumonia: a population-based case-control study. Arch Intern Med. 2007; 167(9):950-955.

9. Breu AC, Allen-Dicker J, Mueller S, Palamara K, Hinami K, Herzig SJ. Hospitalist and primary care physician perspectives on medication management of chronic conditions for hospitalized patients. J Hosp Med. 2014;9(5):303-309.

10. Bondesson A, Eriksson T, Kragh A, Holmdahl L, Midlöv P, Höglund P. In-hospital medication reviews reduce unidentified drug-related problems. Eur J Clin Pharmacol. 2013;69(3):647-655. 